\title{
Renal Function Assessment Is Part of Endovascular Aortic Aneurysm Repair and It's Follow Up
}

\author{
*Corresponding author \\ Arnaud Kerzmann, Department of Cardiovascular and \\ Thoracic Surgery, University Hospital of Liège, Belgium, \\ Email: akerzmann@chuliege.be \\ Submitted: 15 June 2020 \\ Accepted: 23 June 2020 \\ Published: 24 June 2020 \\ ISSN: 2378-9344 \\ Copyright \\ (c) 2020 Arnaud K, et al. \\ OPEN ACCESS
}

\author{
Kerzmann Arnaud ${ }^{1 *}$, Krzesinski Jean-Marie², Sakalihasan Natzi ${ }^{3}$, \\ and Defraigne Jean-Olivier ${ }^{3}$ \\ ${ }^{I}$ Department of Cardiovascular and Thoracic Surgery, University Hospital of Liège, Belgium \\ ${ }^{2}$ Department of Nephrology, University Hospital of Liège, Belgium \\ ${ }^{3}$ Department of Cardiovascular and Thoracic Surgery, University Hospital of Liège, Belgium
}

Endovascular aneurysm repair (EVAR) is the preferred approach for treatment of abdominal aortic aneurysm (AAA) when anatomy is compatible. Early and medium term morbidity and mortality rates are lower with EVAR than open aortic repair (OAR). In the long term EVAR is however associated with higher reinterventions rate and lower survival [1].

Acute kidney injury (AKI) is a common complication after both EVAR and OAR [2]. Based on the Acute Kidney Injury Network (AKIN) and Risk, Injury, Failure, Loss, End-stage (RIFLE) staging systems, AKI is defined as increase in serum creatinine by $\geq 0,3 \mathrm{mg} / \mathrm{dl}$ within 48 hours, or increase in serum creatinine to $\geq 1,5$ times baseline within 7 days, or urine output $<0,5 \mathrm{ml} / \mathrm{kg} / \mathrm{h}$ for 6 hours [3]. The mechanisms of renal damage after EVAR are multiple: contrast induced nephropathy, renal micro-embolization secondary to intra-aortic navigation or device deployment, coverage of accessory renal artery, iatrognic renal artery dissection, ischemia reperfusion syndrome due to lower limb ischemia, hypovolemia, aneurysmal sac inflammatory infiltrate and patient related cardiovascular comorbidities and treatment [4].

The incidence of AKI after EVAR varies from 5 to $18 \%$ [2-4]. In the prospective study of Saratzis and et al. [4], AKI is more prevalent in patients with impaired renal function and hypercholesterolemia. In the Midlands Aortic Renal Injury (MARI) cohort study, age, history of ischaemic heart disease and baseline estimated glomerular filtration rates (eGFR) are predictors of AKI for patients undergoing EVAR [5]. Statius van Eps and al reported in their retrospective study [6] that use of angiotensin II blockers and peri-operative complications are risk factors for AKI after EVAR. Both impaired renal function before EVAR and AKI are associated with cardiovascular morbidity and mortality.

Long term renal function decreases after EVAR. Charles and al reported in their 9 years follow up study yearly drop of eGFR of
$1.7 \mathrm{ml} / \mathrm{min} / 1.73 \mathrm{~m}^{2}$ [7]. The decline seems to be steeper the first year after EVAR [8]. This is associated with lower survival. Age, male sex, diabetes and prior history of ischaemic heart disease are associated with eGFR drop of more than 20\%9 years after EVAR in the study of Charles and al [7]. Statius van Eps and al identified aortic neck diameter, renal artery stenosis $>50 \%$ and AKI as risk factors for occurrence of chronic kidney disease after EVAR [6].

AKI and long term renal function decline are common after EVAR and associated with cardiovascular morbidity and mortality. We have to think about it when young patients are candidate for EVAR. Serum creatinine and urine output have to be checked in EVAR peri-operative period. Measurement of eGFR before treatment and during follow up is needed. It will allow the physicians to reinforce renal protective therapies and to send as early as possible patients to nephrologists [9]. Risk factors for AKI and long term renal function decline after EVAR have to be confirmed. Potential new diagnostic markers, prevention modalities and therapeutic agents have to be evaluated and validated in prospective studies.

\section{REFERENCES}

1. Sakalihasan N, Michel JB, Katsargyris A, Kuivaniemi H, Defraigne JO, Nchimi A, et al. Abdominal Aortic Aneurysms. Nat Rev Dis Primers. 2018; 4: 34 .

2. Castagno C, Varetto G, Quaglino S, Frola E, Scozzari G, Bert F, et al. Acute kidney injury after open and endovascular elective repair for infrarenal abdominal aortic aneurysms. J Vasc Surg. 2016; 64: 928933.

3. Section 2: AKI Definition. Kidney Int Suppl. 2012; 2: 19-36.

4. Saratzis A, Melas N, Mahmood A, Sarafidis P. Incidence of Acute Kidney Injury (AKI) after Endovascular Abdominal Aortic Aneurysm Repair (EVAR) and Impact on Outcome. Eur J Vasc Endovasc Surg. 2015; 49: 534-540. 
OSciMedcentral

5. Saratzis A, Joshi S, Benson RA, Bosanquet D, Dattani N, Batchelder A et al. Acute Kidney Injury (AKI) in Aortic Intervention: Findings From the Midlands Aortic Renal Injury (MARI) Cohort Study. Eur J Vasc Endovasc Surg. 2020; 59: 899-909.

6. Statius van Eps RG, Nemeth B, Mairuhu RTA, Wever JJ, Veger HTC, van Overhagen $\mathrm{H}$, et al. Determinants of Acute Kidney Injury and Renal Function Decline After Endovascular Abdominal Aortic Aneurysm Repair. Eur J Vasc Endovasc Surg. 2017; 54: 712-720.

7. Charles ER, Lui D, Delf J, Sayers RD, Bown MJ, Sidloff D, et al. The
Impact of Endovascular Aneurysm Repair on Long Term Renal Function Based on Hard Renal Outcomes. Eur J Vasc Endovasc Surg. 2019; 58: 328-333.

8. Saratzis A, Bath MF, Harrison S, Sayers RD, Mahmood A, Sarafidis P, et al. Long-Term Renal Function after Endovascular Aneurysm Repair. Clin J Am Soc Nephrol. 2015; 10: 1930-1936.

9. Krzesinski JM, Defraigne JO, Sakalihasan N. How to Reduce the Risk of Acute Kidney Injury in Abdominal Aortic Aneurysm Surgery. The Quest of the Grail. Eur J Vasc Endovasc Surg. 2020; 59: 866-867.

\section{Cite this article}

Halevy S, Gebrewold A, Carella A, Altura BM (2020) HDFx: A Biologic Ameliorates Deep Vein Thrombosis in Two Rodent Animal Models: In-Vivo Microcirculatory Studies and Relation to Human DVT. Ann Vasc Med Res 7(3): 1112. 